\title{
Probabilistic Issue of Reliability for Power Machinery Operating in Coal Fired Power Plants
}

\author{
Janusz Buchta, Andrzej Oziemski, Maciej Pawlik \\ Lodz University of Technology, Institute of Electrical Power Engineering, Lodz, Poland \\ Email: janusz.buchta@p.lodz.pl, andrzej.oziemski@p.lodz.pl
}

Received January 2014

\begin{abstract}
The paper presents results of reliability analysis made for lignite fired $370 \mathrm{MW}$ rated power units installed in the Belchatow Power Plant (Poland). The concept of standardized power unit and the method of a histogram with a set number of observations in each class were applied in a study. The study includes analysis of probability distributions of operation times and repair times for the main power unit components. Empirical probability distribution functions have been identified and their parameters estimated in the study. The final forecast includes an estimation of such reliability measures like expected operation time, expected failure rate, average repair time and expected annual failure duration.
\end{abstract}

\section{Keywords}

Power Unit; Reliability Indices; Probability Distribution Functions of Operation Time and Repair Time

\section{Introduction}

The maintenance practice shows that failure frequency of power unit components is many times higher than other components of the power system (overhead lines, transformers, switchgear, control systems, protections etc.) [1,2]. Intense wear and tear of machine elements as well as the necessity of maintenance works effect routine repairs of power units. The economic impact of power unit shut-down depends mainly on failure frequency, duration of repair and the period of the year in which the shut-down happens. A very important issue is therefore to optimize the values of these quantities.

The main reasons of power machinery failures can be classified as follows:

- Errors in construction and design;

- Material defects;

- Assembly errors;

- Operational wear and tear of material (corrosion, erosion, fatigue, strain, ageing);

- Influence of external conditions (e.g. lack of fuel or water, power system disturbances);

- Mistakes in repairs; 
- Mistakes in machinery operation;

- Insufficient care to keep installation in appropriate technical condition.

The first three groups of mentioned above reasons generally reveal during the early lifetime of power machinery and are removed au courant during the warranty repairs. Reasons belonging to the fourth revealed after a longer period of operation. However, particular attention should be paid to the last three groups of reasons which depend directly on skills of operational staff working in power plant.

\section{Mathematical Basis of the Reliability Estimation of Power Units by the Method of a Histogram}

Power units are complicated thermal and mechanical installations consisting of several components with a different level of redundancy. Basic power unit components (boiler, turbine, generator) are singular. However, an auxiliary devices (ID fans, FD fans, coal mills, feed pumps, condensate pumps, cooling water pumps, ash handling etc.), in order to increase reliability of operation, have overt or latent reserve (structural redundancy).

The characteristic feature of power unit reliability, directly resulting from the redundancy of an auxiliary system is the possibility to appear failures of different kind, i.e. power unit continues operation with its rates, power unit operates with power limitation or power unit must be shut-down. This means that power unit is a multi-stage object in sense of its reliability, on the contrary to the two-stage installations staying in ability or inability to operate, which refers to many power transmission and distribution devices. A multi-stage feature causes that reliability analysis of power unit uses Markov or more complex semi-Markov processes, which form an adequate probabilistic model.

An operational reliability analysis of complex installation is performed using a reliability diagram which layout usually differs from the functional structure of an installation. Reliability diagram maps the impact of any item on the reliability of the entire system. The layout of reliability diagram of power plant is of a mixed type with dominance of serial connections of elements.

Analytical methods for the assessment of power unit reliability based on Markov processes, in which reliability measures for the entire unit are determined from reliability indices of single components, have one important drawback - the lack of a sufficiently large population of analyzed events for most components under consideration.

Since the first of dozen 370 MW units was commissioned in the Bełchatów Power Plant in 1982, a systematic research on power units reliability was initiated by the Institute of Electrical Power Engineering at Lodz University of Technology. The principal target of analyses prepared yearly has been estimation of actual reliability measures of main generating devices of power units. Statistical data files of the successive years of the power plant operation have been systematically complemented and verified. The verification of statistical data consist in elimination of events which are not of random origin (i.e. actively influenced by operation and maintenance staff) and these, which were not qualified as break-downs only because there was enough ready-reserve power during failure.

Using reliability model and computer database worked out for the $370 \mathrm{MW}$ power unit, the most defective elements of the boiler, turbine, generator and auxiliary systems have been identified [3,4]. For selected elements, empirical probability density functions of operation times, repair times have been determined with use of the histogram with a set number of observations in each class.

The histogram is often used in statistical data analysis to illustrate the major features of the distribution of the data in a convenient form. It divides up the range of possible values in a data set into classes. In a classical histogram, each data class spans the interval of the same width. The height of a rectangle drawn above each class is proportional to the number of observations in the class. The shape of the histogram sometimes is particularly sensitive to the number of classes. If the classes are too wide, important information might get omitted. On the other hand, if the classes are too narrow, the meaningful information fades due to random variations and small number of data in each class. The histogram with a constant width of class interval dispossesses a random sample of its statistical attributes. The classical histogram is generally used when dealing with large data sets (more then 100 observations). It's difficult to get such a large data set in a reliability analysis of an engineered system. The method of a histogram with a set number of observations in each class was applied in a presented study instead a classical histogram. On the contrary to a classical histogram, the histogram with a set number of observations in each class preserves all statistical features of data set. This histogram is prepared according to the fol- 
lowing steps:

- $\quad$ sorting numbers in a data set of $n$ observations in an ascending order $t_{1}, t_{2}, t_{3}, \ldots t_{n}$;

- calculating number of classes $r$ by the formula $r=2 \ln (n)$ and rounding down $r$ to the nearest integer;

- calculating number of observations $m$ in each class by the formula $m=n / r$ and rounding down $m$ to the nearest integer;

- assigning all observations to each class in such a way that equal numbers from a data set are included in the same class; therefore the number of observations in the $i$-th class $-n_{i}$ can vary from an assumed value $m$;

- determining the lower and upper bound of each class; for example the upper bound of the $4^{\text {th }}$ class (lower bound of the $5^{\text {th }}$ class) is an arithmetic mean of the greatest number included in the $4^{\text {th }}$ class and the lowest number included in the $5^{\text {th }}$ class;

- calculating the $i$-th class interval width - $\Delta t_{i}$ as the difference of its upper and lower bound; determining the $i$-th class mid value $-\bar{t}_{i}$ as an arithmetic mean of its lower and upper bounds;

- calculating the value of an empirical probability density function by the formula

$$
f^{x}\left(\overline{t_{i}}\right)=\frac{n_{i}}{n \cdot \Delta t_{i}} .
$$

The histogram is completed by drawing a bar for each class.

The main advantage of this histogram is the possibility to apply for the amount of classes $r \geq 7$ and less numerous data sets. If take into account the minimum required amount of observations in a single class $\left(n_{i} \geq 5\right)$, the minimum statistical sample $(n \geq 35)$ is easy to achieve in practice.

Calculation unit was implemented in computer database to identify probabilistic models for empirical distributions of operational times and shut-down times of standardized $370 \mathrm{MW}$ unit. Considering times of failures recorded in a computer database, times to failure and times of failure duration have been calculated. An empirical probability density function $f^{x}(t)$ is compared with the shape of the density function of different theoretical distributions (exponential, Weibull, normal, log-normal [5]), and subjectively best distribution is selected as the one representing the random variable under investigation. The hypothesis thus constructed is verified by means of (Pearson's and Kolmogorov) statistical tests of goodness of fit and only on this basis is the decision made to accept or reject it. All calculations are performed for the standard significance level $\alpha=0.05$.

The Kolmogorov test compares the empirical distribution function with the cumulative distribution function specified by the null hypothesis and tests goodness of fit with this distribution. The hypothesis regarding the distributional form is rejected if the test statistic, $\lambda$, is greater than the critical value $\lambda_{\alpha}$ obtained from a table $\left(\lambda_{0.05}=\right.$ 1.358). Person's chi-square test is an alternative to the Kolmogorov goodness-of-fit test. For the chi-square goodness-of-fit computation, the data are divided into $r$ classes and the test statistic $\chi^{2}$ is calculated. The hypothesis that the data are from a population with the specified distribution is rejected if $\chi^{2}>\chi_{(\alpha, r-c)}^{2}$. The critical value $\chi_{(\alpha, r-c)}^{2}$ is the chi-square percent point function with $r-c$ degrees of freedom and a significance level of $\alpha$. The variable $c$ is the number of estimated parameters for the distribution increased by 1 . For example, for a 3-parameter Weibull distribution, $c=4$.

A Weibull distribution is very popular statistical model in reliability engineering and failure analysis. Operation times are modeled in the study by a Weibull distribution with the probability density function defined as follows:

$$
f(t)=\frac{b}{a} \cdot\left(\frac{t}{a}\right)^{b-1} \cdot \exp \left[-\left(\frac{t}{a}\right)^{b}\right]
$$

where $b$ is the shape parameter and a is the scale parameter.

When $b=1$, then the Weibull distribution reduces to the exponential distribution. Failure duration times are modeled in the study by a log-normal distribution with the probability density function defined as follows:

$$
f(t)=\frac{1}{t \cdot \sigma \cdot \sqrt{2 \pi}} \cdot \exp \left[-\frac{(\ln t-m)^{2}}{2 \cdot \sigma^{2}}\right]
$$

where $m$ is the mean of the natural logarithms of the failure duration times and $\sigma$ the standard deviation of the natural logarithms of the failure duration times. 
Let's consider the histogram shown in Figure 1(a). The data set consists of $n=1520$ observations. The number of class intervals equals $r=14$. According to the table shown in the upper-right corner, each class includes 108 or 109 observations, so that it's a histogram with set number of observations. The width of the $1^{\text {st }}$ class interval is 78 hours, the width of the $2^{\text {nd }}$ class interval -70 hours, the $3^{\text {rd }}$ class -108 hours, etc. The width of each class interval varies from another but the number of observations in each class is nearly the same. The hypothesis of the Weibull distribution has been tested. The shape and scale parameters of the Weibull distribution have been estimated for a given data set. The statistics $\chi^{2}$ and $\lambda$ have been calculated in Pearson's and Kolmogorov tests. The obtained values are less then critical statistics $\chi_{\alpha}{ }^{2}$ and $\lambda_{\alpha}$ respectively, so that both tests accept the Weibull distribution with parameters $a=1391.2$ and $b=0.828$.

Probability density functions $\mathrm{f}(\mathrm{t})$, cumulative distribution functions $\mathrm{F}(\mathrm{t})$ and means $\mathrm{E}(\mathrm{T})$ for considered distributions are presented in Table $\mathbf{1}$.

\section{Histograms of Operation Times and Repair Times for the 370 MW Power Unit, Its Main Components and Selected Elements of the BB-1150 Steam Boiler}

Dozen of lignite fuelled 370 MW rated power units operate currently in Polish power system, located in Belchatow Power Plant, which is the largest power plant of this type in Europe.

The idea of a standardized unit has been introduced into the reliability study of the 370 MW units. All units are homogenous in several respects such as constructional uniformity and similar conditions in which units operate. The standardized unit is the unit in its useful life with stabilized failure rate that substitutes a dozen of power plant units. The assumption that a standardized unit is in its useful life requires excluding a period of an early life from an operation time of respective power units. The concept of a standardized unit allows to obtain an appropriately numerous population of failures not only for a power unit but also for its main generating devices (boiler, turbine, generator).

While establishing the population, the cases of incidental failures that occurred in the initial period of the power plant operation were neglected. It particularly refers to units 1 and 2 during the first three years of the power plant operation. The cases of failures that occurred in first year of operation were also skipped for remaining units. One can state that a higher failure frequency of a 370 MW power units in the initial period of their operation was caused mainly by a design, construction and assembly defects. All these factors are distinctive for an adaptation of new generation power units. Therefore this period was excluded while population of failures for a standardized 370 MW unit was selected.

An investigated probability distribution functions were identified as Weibull distributions with parameter $\mathrm{b}<$ 1 (Figure 1). In emergency states, strong dependence of the time of damage liquidation on the cause of its occurrence was found. Average times of shut-downs, in the case of permanent defects of the installations clearly differ from the times of shut-downs caused by the incorrect operation of the automatic control systems and safety devices, the operational staff mistakes and the like. And so, the values of times are as follows: $41.2 \mathrm{~h}$ and $1.76 \mathrm{~h}$ for the boiler, $59.3 \mathrm{~h}$ and $1.27 \mathrm{~h}$ for the turbine, $78.9 \mathrm{~h}$ and $1.48 \mathrm{~h}$ for the generator, $40.1 \mathrm{~h}$ and $1.95 \mathrm{~h}$ for

Table 1. Probability density functions $f(t)$, cumulative distribution functions $F(t)$ and means $E(T)$ for considered distributions.

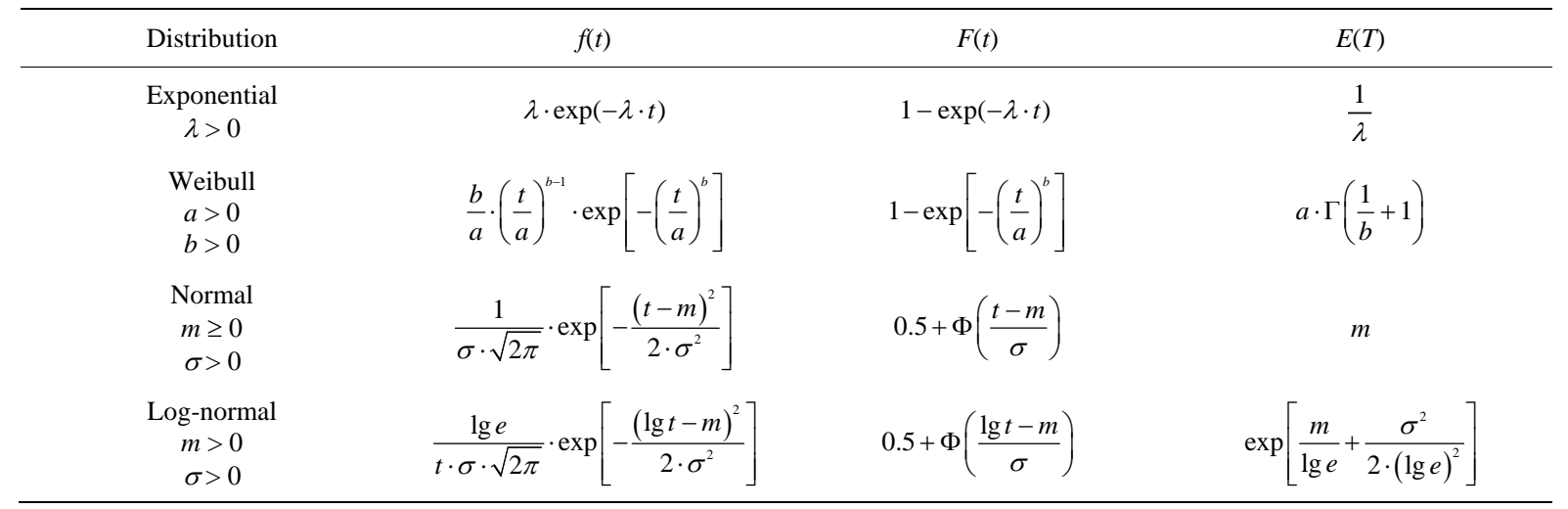

Explanations: $\Gamma$-gamma function, $\Phi$-Laplace's function. 


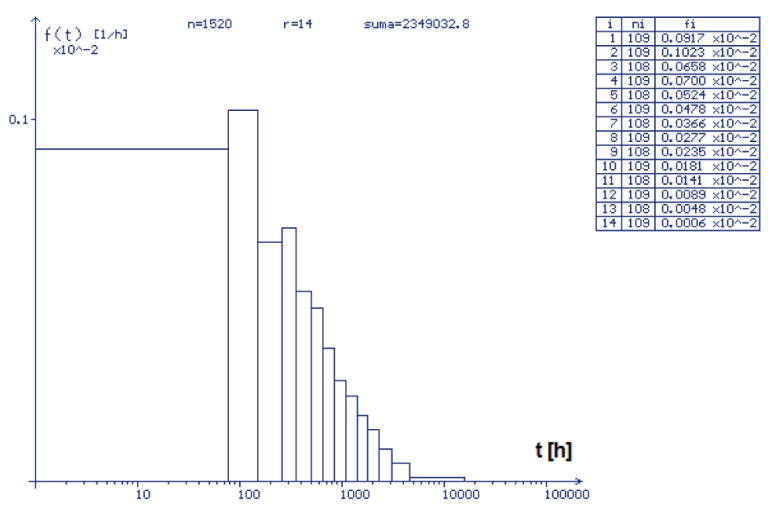

Testing of hypothesis of Weibull distribution: $\mathrm{a}=1391,2, \mathrm{~b}=0,828$ Pearson's test: $\chi^{2}=17,902, \chi_{\alpha}^{2}=19,675$

Kolmogorov test: $\lambda=0,716, \lambda_{\alpha}=1,358$

(a)

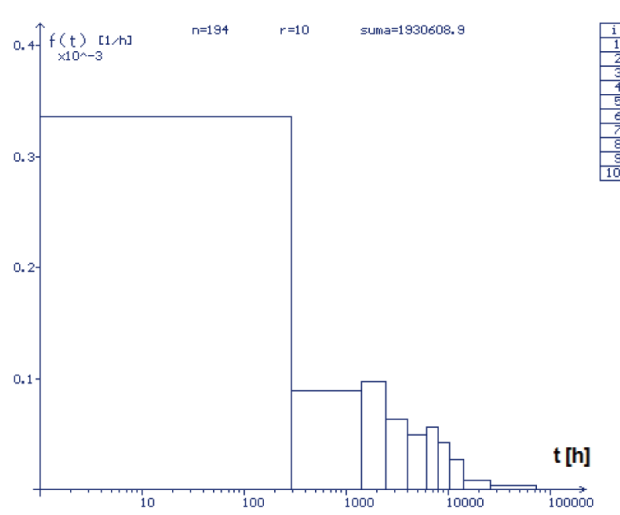

Testing of hypothesis of Weibull distribution: $\mathrm{a}=8703,6, \mathrm{~b}=0,785$ Pearson's test: $\chi^{2}=11,758, \quad \chi_{\alpha}{ }^{2}=14,067$

Kolmogorov test: $\lambda=0,452, \lambda_{\alpha}=1,358$

(c)

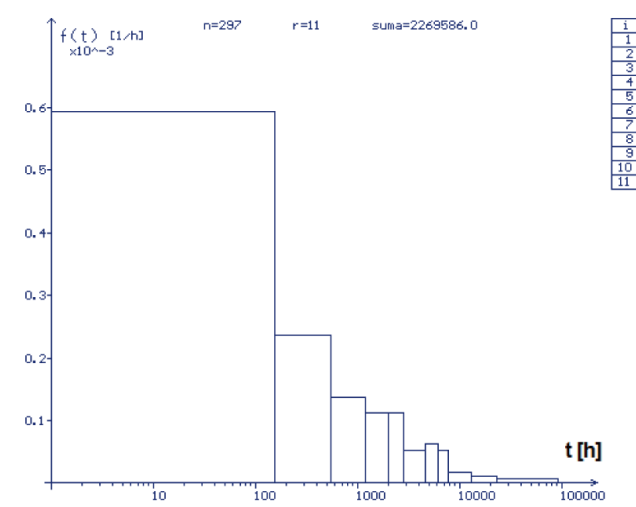

Testing of hypothesis of Weibull distribution: $\mathrm{a}=5674,8, \mathrm{~b}=0,641$ Pearson's test: $\chi^{2}=14,081, \chi_{\alpha}^{2}=15,507$

Kolmogorov test: $\lambda=0,677, \lambda_{\alpha}=1,358$

(b)

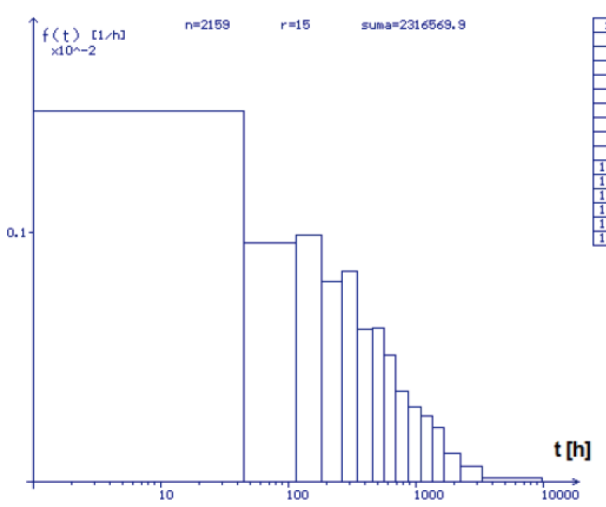

Testing of hypothesis of Weibull distribution: $\mathrm{a}=993,1, \mathrm{~b}=0,806$ Pearson's test: $\chi^{2}=20,872, \chi_{\alpha}^{2}=21,026$

Kolmogorov test: $\lambda=0,646, \lambda_{\alpha}=1,358$

(d)

Figure 1. Probability density of times between failures identified as Weibull's distribution: (a) Boiler BB-1150, (b) Turbine 18K360, (c) Generator GTHW-370, (d) Power unit. Explanations: $\chi^{2}$-Pearson's statistics, $\chi_{\alpha}^{2}$-critical Pearson's statistics for the significance level $\alpha=0.05, \lambda$-Kolmogorov statistics, $\lambda_{\alpha}$-critical value of Kolmogorov statistics $(\alpha=0.05) i$-the class number, $n$ - the sample size, $r$-the number of classes, sum — the total value of all the observations in the sample, $n i-$ the number of observations in the $i$-th class, $f i-$ the value of empirical probability density function for the $i$-th class.

feed water pump, respectively. Thus, it should be concluded that the times of long-term failures and short-term defects belong to two statistically different populations and that it is advisable to study their distributions separately. Generally speaking, the distributions of failure duration times are log-normal distributions (Figure 2). Empirical distribution functions of operation and failure times for each of dozen power units (Figure 3) have been also examined.

\section{The Assessment of Reliability Indices of 370 MW Power Units and Their Components}

By means of estimation, parameters of identified probability distribution functions of operation times and repair times have been calculated for power machinery of 370 MW rated power units under study. Such reliability indices as expected failure rate, expected mean time of the shutdown, expected total time of the shutdowns and expected mean time between failures have been estimated. Table 2 presents expected values of basic reliability indices for power machinery of the standardized 370 MW rated power unit. Similarly, Table 3 presents basic reliability indices for all power units of Belchatow Power Plant.

The statistics shows that failure frequency of the steam boiler BB-1150 is crucial for availability factor 


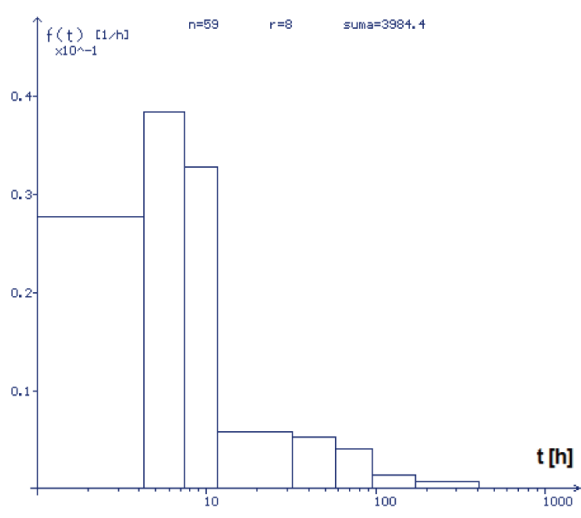

Testing of hypothesis of log-normal distribution: $\mathrm{m}=1,446, \sigma=0,626$ Pearson's test: $\chi^{2}=8,837, \quad \chi_{\alpha}{ }^{2}=11,070$

Kolmogorov test: $\lambda=0,791, \lambda_{\alpha}=1,358$

(a)
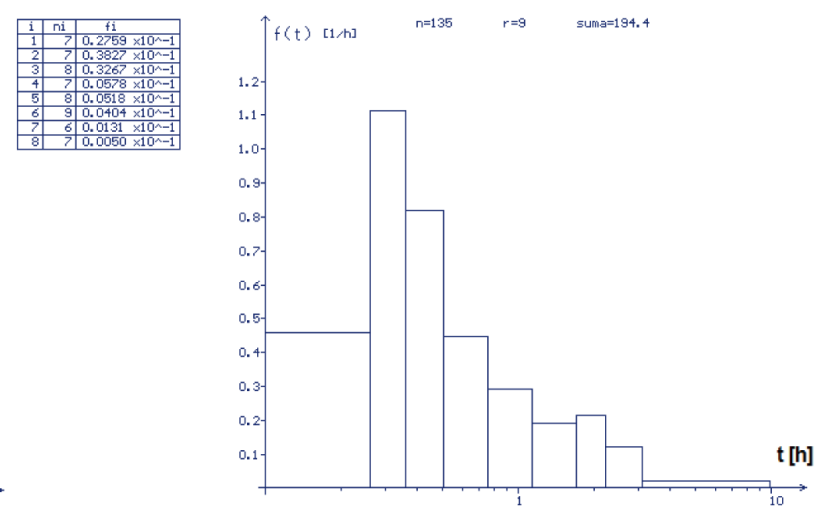

Testing of hypothesis of log-normal distribution: $\mathrm{m}=-0,045, \sigma=0,43$ Pearson's test: $\chi^{2}=11,525, \quad \chi_{\alpha}^{2}=12,592$ Kolmogorov test: $\lambda=0,790, \lambda_{\alpha}=1,358$

(b)

Figure 2. Probability density of failures times identified as log-normal distribution: (a) Generator GTHW-370 (times of long-term failures), (b) Generator GTHW-370 (times of short-term failures).

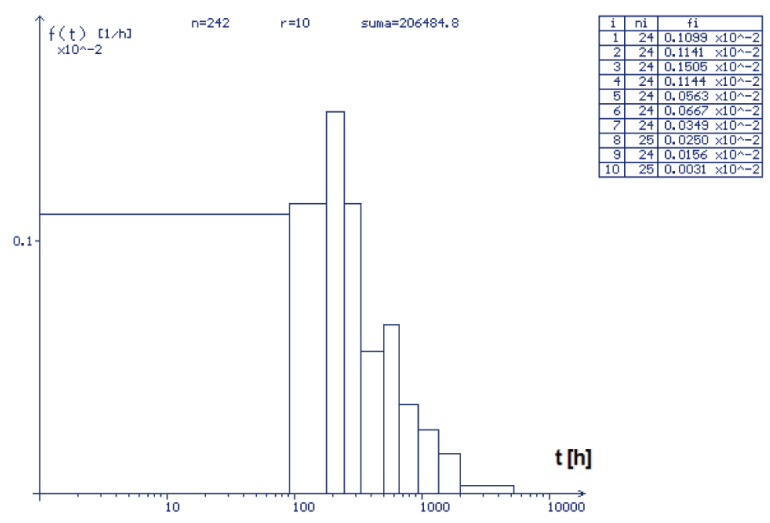

Testing of hypothesis of Weibull distribution: $a=821,5, b=0,927$

$$
\text { Pearson's test: } \chi^{2}=12,127, \chi_{\alpha}^{2}=14,067
$$$$
\text { Kolmogorov test: } \lambda=0,745, \lambda_{\alpha}=1,358
$$

(a)

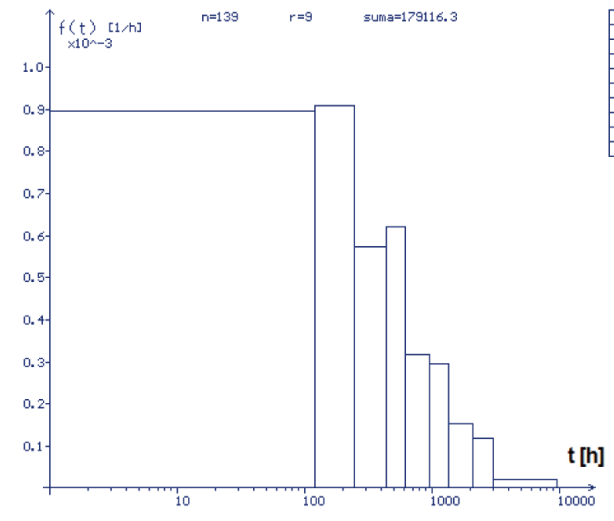

Testing of hypothesis of Weibull distribution: $\mathrm{a}=1224,3, \mathrm{~b}=0,903$ Pearson's test: $\chi^{2}=3,343, \quad \chi_{\alpha}^{2}=12,592$ Kolmogorov test: $\lambda=0,315, \lambda_{\alpha}=1,358$

(b)

Figure 3. Probability density of times between failures identified as Weibull’s distribution: (a) Unit 1, (b) Unit 12.

Table 2. Reliability indices for main power unit components.

\begin{tabular}{ccccc}
\hline Failure location & $\begin{array}{c}\text { Expected failure } \\
\text { rate [1/a] }\end{array}$ & $\begin{array}{c}\text { Mean time of a } \\
\text { shut-down [h] }\end{array}$ & $\begin{array}{c}\text { Total time of } \\
\text { shut-downs [h/a] }\end{array}$ & $\begin{array}{c}\text { Mean time between } \\
\text { failures [h] }\end{array}$ \\
\hline B & 3.90 & 37.8 & 147.3 & 1540 \\
T & 0.76 & 13.0 & 9.89 & 7880 \\
G & 0.69 & 25.0 & 17.3 & 8690 \\
F & 0.30 & 11.0 & 3.24 & 20350 \\
W & 0.16 & 9.3 & 1.52 & 36660 \\
O & 0.46 & 9.0 & 4.1 & 13160 \\
Unit & 5.36 & 31.0 & 166.1 & 1120 \\
\hline
\end{tabular}

Explanations: B-steam boiler and its auxiliaries, T—steam turbine and its auxiliaries, G-generator and its auxiliaries, F-system of feed water pumps, W—system of cooling water and service water pumps, $\mathrm{O}$ - others (including failures of electrical devices). 
Table 3. Reliability indices for dozen power units of Belchatow Power Plant.

\begin{tabular}{ccccc}
\hline Failure location & $\begin{array}{c}\text { Expected failure } \\
\text { rate, [1/a] }\end{array}$ & $\begin{array}{c}\text { Mean time of a } \\
\text { shut-down, [h] }\end{array}$ & $\begin{array}{c}\text { Total time of } \\
\text { shut-downs, [h/a] }\end{array}$ & $\begin{array}{c}\text { Mean time between } \\
\text { failures, [h] }\end{array}$ \\
\hline Unit 1 & 7.05 & 30.1 & 212.4 & 850 \\
Unit 2 & 7.27 & 29.9 & 217.2 & 126.6 \\
Unit 3 & 4.27 & 29.7 & 143.1 & 1210 \\
Unit 4 & 4.96 & 28.8 & 198.3 & 1010 \\
Unit 5 & 5.92 & 33.5 & 157.9 & 1120 \\
Unit 6 & 5.36 & 29.5 & 219.0 & 990 \\
Unit 7 & 6.08 & 36.0 & 171.4 & 1040 \\
Unit 8 & 5.78 & 29.7 & 155.3 & 1240 \\
Unit 9 & 4.83 & 32.2 & 124.8 & 1230 \\
Unit 10 & 4.86 & 25.7 & 125.3 & 1420 \\
Unit 11 & 4.23 & 29.6 & 164.5 & 1290 \\
Unit 12 & 4.67 & 35.3 & & \\
\hline
\end{tabular}

achieved by Belchatow Power Plant. One can state that steam boiler marks out the potential to improve power plant availability factor by reduction of emergency shutdowns with support of optimal scheduling of power plant repairs. Such activities should improve operational durability of the weakest power unit components.

\section{Conclusions}

The security level of power system is determined by its weakest links represented by large power units which failure rates are many times higher than other components of the power system. The lack of knowledge on the reasons and frequency of failures of power machinery makes scheduling plant operation a difficult task as well as optimal scheduling of power plant repairs and enabling of power supply continuity.

Statistical data on operational disturbances that appeared in power plant lifetime have been carried out systematically for Polish power units rated at 370 MW and fuelled with lignite. Having this database, it's possible to analyze the changeability of reliability indices in a long-term perspective and define reasons or effects of defective operation of power machinery. Economically excused failure rates are reasonable measures for durability of power machinery and their components in determination of modernization schedules and rationalization of maintenance and materials management in entire power plants under study.

The final decision on power machinery maintenance should be accepted on the ground of observation of the element intended to repair with use of both diagnostic and prognostic methods. In global sense, power units availability and reliability indices may be useful to determine operating and replacement reserve in power system.

\section{References}

[1] Allan, R.N. and Billinton, R. (1993) Power System Reliability and Its Assessment. Power Engineering Journal.

[2] J. Paska, P. Wójcik, J. Bargiel, W. Goc and P. Sowa, “Data for electric power system calculations," 8th International Conference on Electrical Power Quality and Utilisation, Poland, 1-5 September 2005, pp. 477-482.

[3] Oziemski, A. (2012) Racjonalizacja Gospodarki Remontowej Bloków Energetycznych Opalanych Węglem w Ujęciu Probabilistycznym. Przegląd Elektrotechniczny, 12a, 159-166.

[4] Pawlik, M., Skierski, J. and Oziemski, A. (2001) Zuverlässigkeitsbewertung von Braunkohlegefeuerten Kraftwerksblöcken großer Leistung. XXXIII Kraftwerkstechnischen Kolloquium, Dresden, 141-152.

[5] Kececioglu, D. (2002) Reliability Engineering Handbook. DEStech Publications, Paris. 